\title{
Development of a web-based insulin decision aid for the elderly: usability barriers and guidelines
}

\begin{abstract}
In recent years, researchers have attempted to shift patient decision aids (PDAs) from paperbased to web-based to increase its accessibility. Insulin decision aids help diabetes patients, most of whom are elderly to make an informed decision to start insulin. However, the lack of usability guidelines applicable for such target group causes developers to struggle to answer the challenging question 'How can such web service be made usable, and, ultimately, acceptable and accessible for elderly patients?'. Hence, the purpose of this study is to identify the common usability requirements that may facilitate good practices to empower elderly diabetes patients in utilizing a web-based insulin decision aid for their benefit. We set out an approach to use prototyping and retrospective think-aloud techniques to explore web usability barriers that elderly patients may encounter when using an insulin decision aid web site and use the feedback for improving the prototype. Usability requirements were captured iteratively through scoping, brainstorming, prototype, testing and evaluating. The study suggests that the insights from experts and users are equally important to assure the validity of the identified usability guidelines; they reflect the accessibility needs of the aging community while complementing the key requirements of an insulin decision aid. The study contributes to recommend web usability guidelines backed by a series of expert and user evaluations which could be a proactive resource to improve usability, acceptability and accessibility of online insulin decision aids for elderly with diabetes.
\end{abstract}

Keyword: Elderly; Web usability; User-centered design; Human-computer interaction; Diabetes mellitus; Insulin decision aid 